\title{
Bose-Einstein Condensation in a Constant Magnetic Field
}

\author{
H. Perez Rojas ${ }^{1,2,3}$ and L. Villegas-Lelovski ${ }^{2}$ \\ ${ }^{1}$ High Energy Division, Department of Physics, University of Helsinki, \\ Siltavuorenpenger 20C, FIN-00014, University of Helsinki, Finland \\ ${ }^{2}$ Departamento de Física, Centro de Investigación y de Estudios Avanzados del IPN, \\ Apartado Postal 14-740, 07000 México, D. F., México \\ ${ }^{3}$ Grupo de Física Teórica ICIMAF, \\ Calle E No. 309, Vedado, La Habana 4, Cuba.
}

Received 22 November, 1999

\begin{abstract}
We discuss the occurrence of Bose-Einstein condensation in systems of noninteracting charged particles in three and one dimensions and in presence of an external magnetic field. In the one dimensional, as well as in the magnetic field cases, although not a critical temperature, a characteristic temperature can be found, corresponding to the case in which the ground state density becomes a macroscopic fraction of the total density. The case of relativistic charged scalar and vector particles is studied. The results give support to the existence of superconductivity in extremely strong magnetic fields, and lead to the prediction of superconductive-ferromagnetic behavior in the vector field case, which might be of interest in condensed matter as well as in cosmology. Some features of the magnetization in the early universe are conjectured.
\end{abstract}

\section{Introduction}

Condensation in the ground state seems to be a general property whenever the conditions of quantum degeneracy of the Bose-Einstein gas are satisfied. Quantum degeneracy is usually understood to be achieved when the De Broglie thermal wavelength $\lambda$ is greater that the mean interparticle separation $N^{-1 / 3}$. The remarkable discovery made by Einstein on the Bose distribution was that condensation may occur starting at some critical temperature $T_{c}$ different from zero, which is usually referred as Bose-Einstein condensation (BEC). Condensation of charged particles requires a background of charge of opposite sign to screen the mutual repulsion among particles. We will assume that such background exists.

The condition for BEC to occur in a gas of charged particles placed in a magnetic field is usually borrowed from the case without magnetic field as $\mu=E_{0}$ (where $E_{0}$ is the ground state energy), which can be translated into $\mu=\sqrt{M c^{2} \pm e B \hbar c}$ for the relativistic case ( $\mu^{\prime}=\mu-M c^{2} \pm e B \hbar / M c$ in the nonrelativistic limit). We must cite first Schafroth [2] who proved that for a non-relativistic boson gas, Bose-Einstein condensation (BEC) does not occur in presence of a constant magnetic field. It was implicit in his paper that he considered only the case of not very intense fields. The problem was studied afterwards by May [3], who showed that a phase transition like that in the free gas occurs for $D \geq 5$, and later by Daicic et al [4], [5], who extended the considerations made by [3] to the relativistic high temperature case. Later Toms [6] proved that $\mathrm{BEC}$ in presence of a constant magnetic field does not occur in any number of spatial dimensions, and Elmfors et al [7] who stated that in the $3 D$ case, although a true condensate is not formed, the Landau ground state can be occupied by a large charge density. A simple criterion for $\mathrm{BEC}$ to occur, was given by Toms and Kirsten [6], who concluded that usual BEC can occur only for $d \geq 3$.

There are two ways of characterizing the occurrence of BEC, which are: 1) The existence of a critical temperature $T_{c}>0$ such that $\mu\left(T_{c}\right)=E_{0}$, (This condition is usually taken as a necessary and sufficient condition for condensation; see [9]). Then, for $T \leq T_{c}$, some significant amount of particles starts to condense in the ground state. 2) The existence of a finite fraction of the total particle density in the ground state and in states in its neighborhood at some temperature $T>0$. We refer to 1) as the strong and to 2) as the weak criterion for BEC.

A magnetic field explicitly breaks the spatial symmetry. It is reflected in the wave function of a charged particle moving in it, and also in its spectrum. The spectrum of a charged Fermi or Bose particle in a constant magnetic field indicates this breaking of the sym- 
metry in momentum space: the momentum components perpendicular to the field collapse in a set of discrete Landau quantum states, their energy eigenvalues having infinite degeneracy. A gas of charged particles (either Bosons or Fermions) in presence of very intense magnetic fields populate mainly the Landau ground state $n=0$ and behaves as a one-dimensional gas in the axis $p_{3}$ parallel to the field. For Bosons, it leads to a statistical distribution for fixed temperature $T$ and magnetic field $B$, which depends only on $p_{3}$.

We must bear in mind at this point that the usual properties of second order phase transitions cannot be valid in presence of external fields (see Landau-Lifshitz, [12]). The external field introduces in the Hamiltonian a perturbing operator which is linear in the external field strength, in our case $B$, and in the order operator, in our case $\hat{\mathcal{M}}$, the magnetization. As a result, at any value of $B, \mathcal{M}$ becomes different from zero at any temperature. Thus, $B$ reduces the symmetry of the usual more symmetrical phase, and the difference between the two phases of the usual theory dissapears; the discrete transition point dissapears; the transition is "smoothed out".

Thus, as BEC in 3D has the properties of a second order phase transition, in studying it in presence of a magnetic field, the strong criterium cannot be applied; no critical temperature separating phases of different symmetry exists, and that was confirmed in many of the results of references [2]- [6]. But an order parameter exists at any temperature, the magnetization $\mathcal{M}$, which for large fields and densities, and temperatures small enough (see below) becomes proportional to the ground state density. Actually, the ground state density can be considered also as another order parameter closely related to $\mathcal{M}$ and may become a macroscopic fraction of the total density, not at a definite $T_{c}$, but in some interval of values of the temperature. We may think in the case in which one starts from a gas of charged particles, without magnetic field, below the critical temperature. If a sufficiently strong magnetic field is applied, the particles in the condensate remain condensed, and a critical temperature is not possible to be defined. (It is interesting to mention here that, as shown in ref. [8], there is no critical temperature also in the case of a gas confined to a parabolic potential.)

This paper is a more detailed and complete version of some of the results of two previous ones, [13], [14], in which it was pointed out that Bose-Einstein condensation, in the sense of having a large population in the ground state in a continuous range of temperatures (i.e., no critical temperature exists), may occur in presence of a magnetic field $\mathbf{B}$, for very dense systems at very low temperatures. The existence of discrete Landau quantum states allows this "weak" BEC. In this case, the ground state population is explicitly included in the Bose-Einstein distribution.

The occurrence of Bose-Einstein condensation in a strong magnetic field gives theoretical support to the phenomenon of re-appearance of superconductivity in fields strong enough, and even leads to the prediction of superconductive-ferromagnetic behavior in the vector field case, a phenomenon which would be of especial interest in condensed matter and in cosmology.

The structure of the present paper is as follows. In section II we shall discuss some features of the usual $3 D$ Bose-Einstein condensation, which gives a basis to understand its "weak" occurrence in the one-dimensional case (section III) and in the magnetic field case (section IV). In section $\mathrm{V}$ it is explicitly shown the ferromagnetic behavior of the magnetization of the vector field case. It is also discussed the connection with superconductivity, the existence of low-field and strong-field condensation and the corresponding superconductive behavior, separated by a gap, and the ferromagnetic-superconductive behavior appearing in the vector field case. section VI is devoted to some cosmological considerations, and section VII deals with the conclusions.

\section{The usual Bose-Einstein Condensation}

In order to be self-contained, we recall some results and formulae from [13], [14]. concerning the standard $3 D$ BEC theory of the free Bose gas. We must emphasize that our considerations in the present and next section are essentially concerned with the case of systems far from the thermodynamic limit. We shall use throughout the paper $T$ in energy units, i.e., as the product of the Boltzmann constant $k$ by the absolute temperature; thus, $T=\beta^{-1}$. The chemical potential $\mu=f(N, T)<0$ is a decreasing function of temperature at fixed density $N$, and for $\mu=0$ one gets an equation defining $T_{c}=f_{c}(N)$. For temperatures $T<T_{c}$, as $\mu=0$, the expression for the density gives values $N^{\prime}(T)<N$, and the difference $N-N^{\prime}=N_{0}$ is interpreted as the density of particles in the condensate. The mean interparticle separation is then $l=N^{-1 / 3}$.

In our considerations we will use integrals which, as it is usually done, must be interpreted as approximations of sums over discrete quantum states, which imply not to be working at the thermodynamic limit. For usual macroscopic systems, as the separation between quantum states is $\Delta p=h / V^{1 / 3}$, the approximation of the sum by the integral is quite well justified.

Now, above the critical temperature for condensation

$$
N=4 \pi^{-1 / 2} \lambda^{-3} \int_{0}^{\infty} \frac{x^{2} d}{e^{x^{2}+\bar{\mu}}-1}=\lambda^{-3} g_{3 / 2}(z),
$$

where $\bar{\mu}=-\mu / T(>0), x=p / p_{T}$ is the relative momentum, $p_{T}=\sqrt{2 m T}$ the characteristic thermal momentum, and $\lambda=h /(2 \pi m T)^{1 / 2}$ the de Broglie thermal wavelength. The function $g_{n}(z)$ is easily defined (see 
Pathria [10]), as a function of the fugacity $z=e^{\mu / T}$, At $T=T_{c}$, we have $g_{n}(1)=\zeta(n)$, and $g_{3 / 2}(1)=\zeta(3 / 2)$, and the density is $N_{c}=\zeta(3 / 2) / \lambda^{3}$, or in other words, $N \lambda^{3}=\zeta(3 / 2) \simeq 2.612$. Here we must point out that if the particle density is written as a sum over quantum states

$$
N=\sum_{i}\left(z^{-1} e^{\frac{E_{i}}{T}}-1\right)^{-1},
$$

for strictly $z=1(\mu=0)$, the ground state term diverges. (In field theoretical language, it is due to an infrared divergence of the one-particle Green function at $\mu=0)$. This divergence usually serves as an argument [10] to indicate the occurrence of Bose-Einstein condensation, and actually we must take $\mu=0_{+}$. However, in considering temperatures below $T_{c}$, it is a very good approximation to take $\mu=0$ [10], [12], and thus the ground state density is described by the expression

$$
N_{0}=N\left[1-\left(\frac{T}{T_{c}}\right)^{3 / 2}\right]
$$

It leads to conclude that for $T=T_{c}, N_{0}=0$. This seems to be rather inconsistent with our previous analysis about the limit of (2) for $\mu \rightarrow 0$, and one expect that (3) is not exact, but must contain terms of order smaller than $N$, accounting for the ground state density at $T=T_{c}$. One can proceed in a different way, by keeping in mind the temperature Green's function formalism [15], which in the non-relativistic infinite volume limit leads to $N=\int d^{3} \mathbf{p} G(p)$. By considering a finite volume, $N$ results as a sum over degenerate energy states, the degeneracy factor being proportional to $p^{2} \Delta p / h^{3}$. This leads to a finite contribution in the limit $p=0$, since the infrared divergence of the Green function at the pole $\mu-E_{0}$ is canceled by the factor $p^{2}$, leading to a non-zero but finite density at the ground state at $T=T_{c}$, as will be seen below.

Before doing that, it is interesting to investigate in detail the particle density in relative momentum space

$$
f_{3}(x, \bar{\mu})=\frac{x^{2}}{e^{x^{2}+\bar{\mu}}-1} .
$$

By calculating the first and second derivatives of this function, we find that for $\bar{\mu} \neq 0$ it has a minimum at $x=0$ and a maximum at $x=x_{\mu}$ where $x_{\mu}$ is the solution of $e^{x^{2}+\bar{\mu}}=1 /\left(1-x^{2}\right)$.

In this sense $f(x, \bar{\mu})$ has a similar behavior than the Maxwell-Boltzmann distribution of classical statistics. But as $\bar{\mu} \rightarrow 0$, also $x_{\mu} \rightarrow 0$, and the maximum of the density, for strictly $\bar{\mu}=0$, is located at $x=0$. The convergence to the limit $x=0$ is not uniform. A finite fraction of the total density falls in a vicinity of the ground state. This behavior is illustrated in Fig. 1.

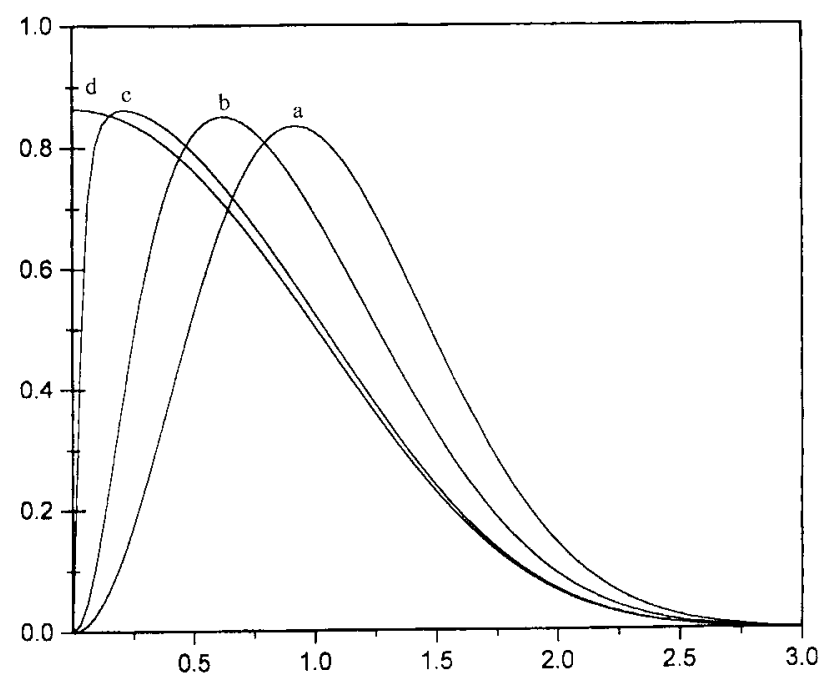

Figure 1. The density in momentum space of the Bose gas for $T>T_{c}$ has a minimum at $p_{3}=0$ and a maximum at some $p_{3} \neq 0$. As temperature is decreased, the maximum approaches to the value $p_{3}=0$ and at $T=T_{c}$ it is rigorously on it, the minimum disappears. The curves a, b, c, d correspond respectively to $\bar{\mu}=1,0.1,0.001$ and 0 .

If we go back and substitute the original integral over momentum by a sum over shells of quantum states of momentum (energy states), we can write for the critical value $\bar{\mu}=0$,

$$
N_{c}=\frac{4 \pi}{h^{3}} \sum_{i=0}^{\infty} \frac{p_{i}^{2} \Delta p}{e^{p_{i}^{2} / 2 M T}-1} .
$$

Taking $\Delta p \simeq h / V^{1 / 3}$, where $V$ is the volume of the vessel containing the gas, the contribution of the first term $p_{0}=0$ of the ground state density is $N_{0}=\frac{4}{V^{1 / 3} \lambda^{2}}$. We have thus a fraction of

$$
N_{0} / N_{c}=4 \lambda \zeta(3 / 2) / V^{1 / 3}=4 \zeta(3 / 2)^{-2 / 3} / \mathcal{N}^{1 / 3}
$$

particles in the ground state, (where $\mathcal{N}=N V$ is the total number of particles) which is the most populated, as described by the statistical distribution, at $T=T_{c}$. A numerical estimation for one litre of $\mathrm{He}$ gas leads to $N_{0} / N \simeq 10^{-6}$. In quantum states in a small neighborhood of the ground state, the momentum density has slightly lower values. Thus, at the critical temperature for BEC, there is a set of states close to the ground state, having relative large densities. Although this result was obtained using exactly $\bar{\mu}=0$, which according to (2) implies an infinite ground state population, it represents an improvement compared with $N_{0}=0$ for $T=T_{c}$.

Eq. (5) indicates an interesting relation: the larger the separation between quantum states, (i.e., the smaller the volume) the larger the population of the ground state at the critical temperature. In the thermodynamic limit the quantum states form a continuum, and (5) has no meaning. However, most systems of physical interest, in laboratory as well as in astrophysical contexts, have finite $V$ and $\mathcal{N}$. 
We conclude that for values of $T<T_{c}$, the curve describing the density in momentum space flattens on the $p$ (or $x$ ) axis, and its maximum decreases also. As conservation of particles is assumed, we get the ground state density by adding to $f(x)$ the quantity $2 N\left[1-\left(T / T_{c}\right)^{3 / 2}\right] \theta\left(T_{c}-T\right) \lambda^{3} \delta(x)$ as an additional density. As $T \rightarrow 0$, the density in the ground state increases at the expense of non-zero momentum states. This leads to the usual Bose-Einstein condensation. We must remark that in that case, for values of $T$ smaller but close to $T_{c}$, both the weak and the strong criteria are satisfied.

\section{The one-dimensional case}

The condensation in $1 \mathrm{D}$ is interesting in itself, since there is an incresing interest to study condensation in systems of lower dimensionality than 3D (we discussed the ideal 2D gas case in [14]), taking into account its possible experimental realization (even the problem of superfluidity in quasi 1D systems is currently discussed in the literature, see [11]). Here we will be interested on it thinking in its connection with the magnetic field case. For $D=1$, there is reduced symmetry with regard to the $3 \mathrm{D}$ case, and no critical temperature is expected to occur. In this case, the mean interparticle separation is $l=N^{-1}$. The density in momentum space coincides with the Bose-Einstein distribution, $f_{1}(p, T, \mu)=\left(e^{p^{2} / 2 M T-\mu / T}-1\right)^{-1}$. This function has only one extremum, a maximum, at $p=0$. We have the expression for the density of particles as

$$
N=2 \lambda^{-1} \pi^{-1 / 2} \int_{0}^{\infty} \frac{d x}{e^{x^{2}+\bar{\mu}}-1}=\frac{1}{\lambda} g_{1 / 2}(z) .
$$

We have thus that $N \lambda=g_{1 / 2}(z)$. The fact that $g_{1 / 2}(z)$ diverges as $z \rightarrow 1$ indicates an enhancement of the quantum degeneracy regime. But this fact actually means that $\mu$ is a decreasing function of $T$ for $N$ constant, and for very small $\bar{\mu}$ one can write, approximately

$$
N \simeq 2 \lambda^{-1} \pi^{-1 / 2} \int_{0}^{x_{0}} \frac{d x}{x^{2}+\bar{\mu}} \simeq \frac{\pi^{1 / 2}}{\bar{\mu}^{1 / 2} \lambda},
$$

where $x_{0}=p_{0} / M T, p_{0}$ being some characteristic momentum $p_{0} \gg p_{T}$. Thus, $\bar{\mu}$ does not vanish at $T \neq 0$, and for small $T$ it is approximately given by $\bar{\mu}=$ $\pi / N^{2} \lambda^{2}$. Then $\bar{\mu} \ll x_{0}$ implies $p_{0} \gg 2 \pi^{2} M^{2} T^{2} / N^{2} h^{3}$, and it is fulfilled if $T / N \rightarrow 0$.

By substituting the last expression for $\bar{\mu}$ back in (7), one has

$$
N \simeq 2 \lambda^{-1} \pi^{-1 / 2} \int_{-x_{0}}^{x_{0}} \frac{d x}{x^{2}+\gamma^{2}},
$$

where $\gamma=\pi^{1 / 2} / N \lambda$. Due to the properties of the Cauchy distribution, one can find that one half of the density is in the interval $[0, \gamma]$,

$$
\frac{1}{2} N=2 \lambda^{-1} \pi^{-1 / 2} \int_{0}^{\gamma} \frac{d x}{x^{2}+\gamma^{2}}
$$

We can also find

$$
f_{1}(p, T, N)_{\gamma \rightarrow 0} \simeq \frac{2 N \lambda}{\pi^{1 / 2}} \delta(p)
$$

Thus, for small $\gamma$ one-half of the density is concentrated in the interval of momentum $[-\gamma, \gamma]$. To precise figures, we shall define $\gamma^{\prime}=\gamma p_{T}$, where $p_{T}=\sqrt{2 \pi M T}$ is the de Broglie momentum, to give the proper dimensions, and compare it with the ground state quantum cell, $2 \pi \hbar / L$, where $L$ is the one dimensional volume of our system. For $2 \pi \hbar / L \gg \gamma^{\prime}$, most of the system is in the ground state. This means that the adimensional phase space density is much greater than the "volume" of the system measured in $\lambda$ units, $N \lambda \gg L / \lambda$.

For $2 \pi \hbar / L \simeq \gamma^{\prime}, N \lambda \sim L / \lambda$, and we can define a temperature $T_{d}=N h^{2} / 2 \pi^{3 / 2} m L$ which, although not being a critical temperature for a phase transition, establishes the order of magnitude of $T$ in which the ground state density is a macroscopic fraction of the total density $N$. Thus, in the present case we have a weak condensation.

\section{The magnetic field case}

By assuming as in [7] a constant microscopic magnetic field $B$ along the $p_{3}$ axis (the external field is $H^{e x t}=B-4 \pi \mathcal{M}(B)$, where $\mathcal{M}(B)$ is the magnetization), for $e B \hbar / m c \gg T$, all the density can be taken as concentrated in the Landau state $n=0$, the maximum of the density in momentum space is just at the point $x=0$ even for $\mu^{\prime} \neq 0$; thus, we conclude that without any critical temperature in that case a finite fraction of the density is found in the ground state.

The energy of a charged particle in a magnetic field in the non-relativistic case is $p_{3}^{2} / 2 M+e B \hbar\left(n+\frac{1}{2}\right) / M c$. Here we name $\mu_{1}=e B \hbar / 2 M c-\mu$ as the effective chemical potential. By defining the elementary cell as $v=\lambda h c / e B$ we can write

$$
\begin{aligned}
N= & \frac{1}{v} \int_{0}^{\infty} \frac{d x}{e^{x^{2}+\bar{\mu}_{1}}-1} \\
& \frac{1}{v} g_{1 / 2}(z)=\frac{1}{v} \sum_{m=1}^{\infty} \frac{e^{-\mu_{1} m \beta}}{m^{1 / 2}}
\end{aligned}
$$

By introducing the continuous variable $x=T m$ and by writing $T \sum_{n=1}^{\infty} \rightarrow \int_{0}^{\infty}$ we get

$$
N=\frac{\pi^{1 / 2}}{v \bar{\mu}_{1}^{1 / 2}}
$$


and in a similar way as before one can get for the relative population in the ground state

$$
\frac{N_{0}}{N}=\frac{\lambda}{L \bar{\mu}_{1}^{1 / 2}}
$$

Here $L$ is the characteristic dimension of the system (along the magnetic field). We observe that $N_{0} / N$ increases with decreasing $\bar{\mu}_{1}$, i.e., as temperature decreases. The limit, which is not transparent from (12) must be unity, as we shall see below. We conclude that the density in momentum space in the magnetic field case concentrates in a (decreasing with $\mathrm{T}$ ) narrow peak around the ground state $p_{3}=0$ (Fig. 2).

We turn to the relativistic case. The thermodynamic potential for a gas of charged scalar particles placed in the magnetic field is,

$$
\Omega_{s}=\frac{e B}{4 \pi^{2} \hbar^{2} c \beta} \sum_{n=0}^{\infty} \int_{-\infty}^{\infty} d p_{3}\left[\ln \left(1-e^{-\left(\epsilon_{q}-\mu\right) \beta}\right)\left(1-e^{-\left(\epsilon_{q}+\mu\right) \beta}\right)+\beta \epsilon_{q}\right]
$$

where $\epsilon_{q}=\sqrt{p_{3}^{2} c^{2}+M^{2} c^{4}+2 e B \hbar c\left(n+\frac{1}{2}\right)}$, the last term in (1) accounts for the vacuum energy and $\mu$ is the chemical potential. For a vector field the one-loop thermodynamic potential is

$$
\begin{aligned}
\Omega_{v}= & \frac{e B}{4 \pi^{2} \hbar^{2} c \beta} \int_{-\infty}^{\infty} d p_{3}\left[\ln \left(1-e^{-\left(\epsilon_{0 q}-\mu\right) \beta}\right)\left(1-e^{-\left(\epsilon_{0 q}+\mu\right) \beta}\right)+\beta \epsilon_{q}\right] \\
& +\frac{e B}{4 \pi^{2} \hbar^{2} c \beta} \sum_{n=0}^{\infty} \beta_{n} \int_{-\infty}^{\infty} d p_{3}\left[\ln \left(1-e^{-\left(\epsilon_{q}-\mu\right) \beta}\right)\left(1-e^{-\left(\epsilon_{q}+\mu\right) \beta}\right)+\beta \epsilon_{q}\right]
\end{aligned}
$$

where $\beta_{n}=3-\delta_{0 n}, \epsilon_{0 q}=\sqrt{p_{3}^{2} c^{2}+M^{2} c^{4}-e B \hbar c}, \epsilon_{q}=\sqrt{p_{3}^{2} c^{2}+M^{2} c^{4}+2 e B \hbar c\left(n+\frac{1}{2}\right)}$.

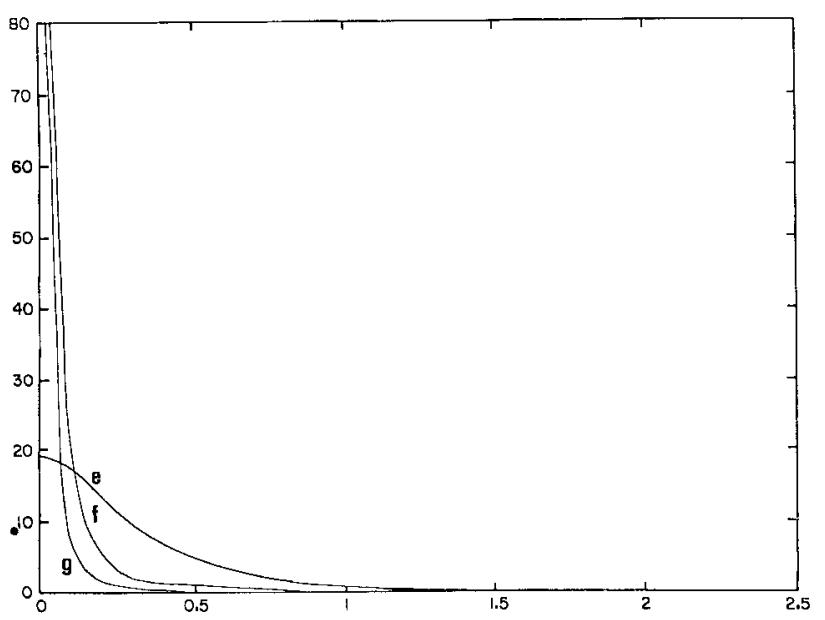

Figure 2. In the magnetic field case, for $e B \hbar / m c T \gg 1$, the system is confined to the $n=0$ Landau quantum state. The maximum of the density in the momentum component along the magnetic field is located at zero momentum at any temperature, and for very low $T$, it has a peaked $\delta$-like form. Here curves d, e, f correspond to $\bar{\mu}=1,0.1$ and 0.001 .

The mean density of particles minus antiparticles (average charge divided by $e$ ) is given by $N_{s, v}=$ $-\partial \Omega_{s, v} / \partial \mu$.

We have explicitly

$$
N_{s}=\frac{e B}{4 \pi^{2} \hbar^{2} c} \sum_{0}^{\infty}\left[\int_{-\infty}^{\infty} d p_{3}\left(n_{p}^{+}-n_{p}^{-}\right)\right] .
$$

where $n_{p}^{ \pm}=\left[\exp \left(\epsilon_{q} \mp \mu\right) \beta-1\right]^{-1}$.

We take the expression fro the vector field case from the Weinberg- Salam Lagrangian in a medium in a magnetic field [16]. We have,

$$
\begin{aligned}
N_{v}= & \frac{e B}{4 \pi^{2} \hbar^{2} c}\left[\int_{-\infty}^{\infty} d p_{3}\left(n_{0 p}^{+}-n_{0 p}^{-}\right)\right] \\
& +\frac{e B}{4 \pi^{2} \hbar^{2} c} \sum_{0}^{\infty} \beta_{n}\left[\int_{-\infty}^{\infty} d p_{3}\left(n_{p}^{+}-n_{p}^{-}\right)\right](16)
\end{aligned}
$$

where $n_{0 p}^{ \pm}=\left[\exp \left(\epsilon_{0 q} \mp \mu\right) \beta-1\right]^{-1}, \quad \epsilon_{0 q}=$ $\sqrt{p_{3}^{2} c^{2}+M^{2} c^{4}-e B \hbar c}$, and $\beta_{0 n}=3-\delta_{0 n}$.

For $T \rightarrow 0, \mu \rightarrow M_{ \pm} c^{2}$ (we named $M_{ \pm}=$ $\left.\sqrt{M^{2} \pm e B \hbar / c^{3}}\right)$, the population in Landau quantum states other than $n=0$ vanishes (this was shown in [1]) and the density for the $n=0$ state is infrared divergent. We expect then most of the population to be in the ground state, since for small temperatures $n_{0 p}^{-}$ is vanishing small and $n_{0 p}^{+}$is a bell-shaped curve with its maximum at $p_{3}=0$. We will proceed as in [16] and call $p_{0}\left(\gg \sqrt{-2 M \mu^{\prime}}\right)$ some characteristic momentum. We have then, by assuming $-\mu^{\prime} \ll T$, for the net density in a small neighborhood of $p_{3}=0$, 


$$
\begin{aligned}
N_{0 s, v}= & \frac{e B T}{2 \pi^{2} \hbar^{2} c} \int_{0}^{p_{0}} \frac{d p_{3}}{\sqrt{p_{3}^{2} c^{2}+M^{2} c^{4} \pm e B \hbar c}-\mu}-\int_{0}^{p_{0}} \frac{d p_{3}}{\sqrt{p_{3}^{2} c^{2}+M^{2} c^{4} \pm e B \hbar c}+\mu} \\
& \simeq \frac{e B T}{2 \pi^{2} \hbar^{2} c^{2}} \int_{0}^{p_{0}} \frac{\left(M_{ \pm} c^{2}+\mu\right) d p_{3}}{p_{3}^{2} c^{2}+M_{ \pm}^{2} c^{4}-\mu^{2}}-\int_{0}^{p_{0}} \frac{\left(M_{ \pm} c^{2}-\mu\right) d p_{3}}{p_{3}^{2} c^{2}+M_{ \pm}^{2} c^{4}-\mu^{2}} \\
& =\frac{e B T}{4 \pi \hbar^{2} c}\left[\sqrt{\frac{M_{ \pm} c^{2}+\mu}{M_{ \pm} c^{2}-\mu}}-\sqrt{\frac{M_{ \pm} c^{2}-\mu}{M_{ \pm} c^{2}+\mu}}\right] \sim \frac{e B T}{4 \pi \hbar^{2} c} \sqrt{\frac{2 M_{ \pm}}{-\mu^{\prime}}}
\end{aligned}
$$

where $N_{s, v}=N_{0 s, v}+\delta N_{s, v}$ and $\delta N_{s, v}$ is the density in the interval $\left[p_{0}, \infty\right]$. Actually $\delta N_{s, v}$ is negligibly small and $N_{s, v}^{0} \simeq N_{s, v}$. We observe that the contribution of antiparticles can be neglected as $\mu \rightarrow M_{ \pm}$. Thus (17) leads to

$$
\mu^{\prime}=-\frac{e^{2} B^{2} T^{2} M_{ \pm}}{8 \pi^{2} N_{0 s, v}^{2} \hbar^{4} c^{2}}
$$

We observe that $\mu^{\prime}$ is a decreasing function of $T$ and vanishes for $T=0$, where the "critical" condition $\mu=M_{ \pm} c^{2}$ is reached. As shown below, in that limit the Bose-Einstein distribution degenerates in a Dirac $\delta$ function, which means to have all the system in the ground state $p_{3}=0$. To see this, we shall rewrite the momentum density of particles around the ground state $p_{3}=0, n=0$ approximately as

$$
\begin{aligned}
n_{0}\left(p_{3}\right)= & \frac{T}{\frac{p_{3}^{2}}{2 M_{ \pm}}+\frac{e^{2} B^{2} T^{2} M_{ \pm}}{8 \pi^{2} \hbar^{4} c^{2} N_{0 s, v}^{2}}} \\
& =\frac{4 \pi \hbar^{2} c N_{0 s, v}}{e B} \cdot \frac{\gamma}{p_{3}^{2}+\gamma^{2}}
\end{aligned}
$$

where

$$
\gamma=\frac{e B T M_{ \pm}}{2 \pi \hbar^{2} c N_{0 s, v}}=\frac{p_{T}}{v N_{0 s, v}}=\sqrt{-2 M_{ \pm} \mu^{\prime}}
$$

where $p_{T}=\sqrt{2 \pi M_{ \pm} T}$ is the thermal momentum, $v=h c \lambda / e B$ the elementary volume cell, $\lambda=h / p_{T}$ being the De Broglie thermal wavelength (observe that $v$ decreases with increasing $B$ ). We have approximated the Bose-Einstein distribution by one proportional to a Cauchy distribution, having its maximum $\sim \gamma^{-1}$ for $p_{3}=0$. We have that $\gamma \rightarrow 0$ for $T \rightarrow 0$, but for small fixed $T, \gamma$ also decreases as $v N_{0 s, v}$ increases. We remind that in the zero field case, the condensation condition demands $N \lambda^{3}>2.612$.

As a property of the Cauchy distribution one can write approximately,

$$
\frac{1}{2} N_{0 s, v}=\frac{e B}{4 \pi^{2} \hbar^{2} c} \int_{-\gamma}^{\gamma} n_{0}\left(p_{3}\right) d p_{3} .
$$

Thus, approximately one half of the total density is concentrated in the narrow strip of width $2 \gamma$ around the $p_{3}=0$ momentum. It results that for densities and magnetic fields large enough, if we choose an arbitrary small neighborhood of the ground state, of momentum width $2 p_{30}$, one can always find a temperature $T>0$ small enough such that $\gamma \ll p_{30}$ and (17) and (20) are satisfied. The condensate appears and it is described by the statistical distribution. Graphs of $n_{0}\left(p_{3}\right)$ for some values of $\bar{\mu}$ are shown in Fig. 2. We have also

$$
\lim _{\gamma \rightarrow 0} n_{0}\left(p_{3}\right)=4 \pi^{2} \hbar^{2} c \frac{N_{s, v}}{e B} \delta\left(p_{3}\right) .
$$

Or equivalently,

$$
N_{s, v}=\lim _{\gamma \rightarrow 0} \frac{e B}{4 \pi^{2} \hbar^{2} c} \int_{-\infty}^{\infty} n_{0}\left(p_{3}\right) d p_{3} .
$$

Thus, if $T \rightarrow 0$, all the density $N_{s, v}$ falls in the condensate, as occur in the zero field case, but here the total density is described explicitly by the integral in momentum space.

One cannot fix any (small) value for $\gamma$ at which the distribution starts to have a manifest $\delta\left(p_{3}\right)$ behavior; and there is no definite value of critical temperature for condensation to start to be constrained essentially in the ground state. But the confinement in the $n=0$ Landau state occurs if the ratio $e B / T$ is large enough to make the average occupation numbers in excited Landau states negligibly small as compared to the ground state. The confinement to the $p_{3}=0$ momentum state along the field, for a given $B$, is determined by the ratio $T / N$. If it is small enough to make $\gamma \ll 2 \pi \hbar / L$ where $L$ is the characteristic length of the system along the magnetic field the condensation takes place essentially in the ground state (these conditions may be achieved 
in non-relativistic systems e. g., for masses $\sim 10^{-27} \mathrm{~g}$, fields of order $10^{6}$ Gauss, $T=10^{-2 \circ} \mathrm{K}$ and $L \sim 1 \mathrm{~cm}$; for Kaons in a neutron star, assuming $T=10^{8} \mathrm{~K}$, $N=10^{39}, B=10^{10}$ Gauss, $L \sim 10 \mathrm{Km}$, it results $\gamma \simeq 10^{-29}$ and $\gamma \ll 2 \pi \hbar / L \simeq 10^{-33}$. One half of the total density can be assumed then to be distributed among $10^{4}$ states; i.e., the ground state density is $10^{35}$, which is a macroscopic number ), The characteristic order of magnitude of the temperature for having a macroscopic fraction of the total density in the ground state, if $L$ is the dimension of the system parallel to the field, is given by $L / \lambda \simeq v N$, or

$$
T_{d} \simeq \frac{4 \pi^{2} \hbar^{3} c N}{e B M_{ \pm} L} .
$$

We observe that for given $N / T$, the quantity $\gamma$ increases with $B$, that is, the increasing field tends to spread the distribution along $p_{3}$, or in other words, to enlarge the density in states close to the groud state, and if $T \simeq T_{d}$, condensation in states neighbor to the ground state become also significant. But for $T \ll T_{d}$, we have almost all the particles in the ground state $n=0, p_{3}=0$, and a true condensate exists.

We return to expression (17). It is easy to obtain the infrared contribution to the thermodynamic potential as

$$
\Omega=\frac{e B T}{4 \pi \hbar^{2} c} \sqrt{M_{ \pm}^{2}-\mu^{2}}
$$

and from $\Omega$, we get the energy $U=T \partial \Omega / \partial T-\Omega$, and the specific heat, which as $T \rightarrow 0$ tends to the constant value,

$$
c_{V}=\frac{2 e^{2} B^{2} M_{ \pm} k^{2}}{4 \pi^{2} \hbar^{4} c^{2} N_{0 s, v}}
$$

where $k$ is the Boltzmann constant.

\section{Magnetization and supercon- ductivity}

For the vector field case, the magnetization in the condensation limit is positive since all the system is in the Landau $n=0$ state, and

$$
\begin{aligned}
\mathcal{M}= & -\frac{\partial \Omega}{\partial B} \\
& =\frac{e^{2} B}{8 \pi^{2}} \int_{\infty}^{\infty} \lim _{\gamma \rightarrow 0} n_{0}\left(p_{3}\right) d p_{3} \\
& =\frac{e N_{v} \hbar}{2 M_{-} c} .
\end{aligned}
$$

and we have that the condensate of vector particles leads to a true ferromagnetic behavior. In particular, if we write $H_{c}^{e x t}=0$, we get $B=\mathcal{M}(B)$ as the condition for spontaneous magnetization to occur. If we started at high temperature $T$, where the system have a diamagnetic contribution coming from the distribution in Landau states, $n=1,2 .$. , and a paramagnetic effect due to the spin coupling, we observe that by lowering $T$ gradually, the system ends in a true ferromagnetic state. A phase transition without having a critical temperature, as the "diffuse" ones [17], has taken place.

Usual superconductivity may be understood in principle as a manifestation of BEC of Cooper pairs, which are spin zero fields. In connection to it Schafroth [2] in his study of the analogy between Bose condensation of scalar charged particles and superconductivity, found a critical applied field $B_{s}=e N_{c s} / 2 M_{+} c$, (where $N_{c s}=N_{s}\left[1-\left(T / T_{c}\right)^{3 / 2}\right], N_{s}$ is the total density and $T_{c}$ the critical temperature for normal condensation), such that the magnetization is equal and opposite to the applied field. In this case when the condensate is contained in some body limited by a surface, and the system is placed in an external magnetic field, it reacts by creating a surface magnetization (Meissner effect) which leads to a magnetic field equal and opposite to the applied one. The charges inside does not feel the external field and remain in the condensate. For field intensities $B>B_{s}$ the (zero field) condensate is destroyed. Obviously, this is the case of not very intense fields, in which the system of charges at the surface are distributed on a large set of Landau states producing a magnetization which is equal and opposite to the external field, keeping the particles inside in free field states. But as the magnetic field grows some of the population inside starts to leave the ground state, and for larger field intensities, the condensate dissapears. However, if the magnetic field increases enough, all the system falls in the $n=0$ Landau ground state, and if the relation $N / T$ is large enough, the condensation becomes manifest again. By calculating $\mathcal{M}_{s}=-\partial \Omega / \partial B$, as the density is (21), we have

$$
\mathcal{M}_{s}=-\frac{e N_{s} \hbar}{2 M_{+} c}
$$

Thus, for growing fields starting from zero, we have usual condensation (and superconductivity), up to the Schafroth's critical field. Then appears a gap where condensation and superconductivity are not observable, and for field and $N / T$ large enough condensation (and superconductivity) reappears.

The phenomenon of condensation of scalar and vector charged particles in strong magnetic fields may have, thus, especial interest in connection with condensed matter physics and cosmology (the early universe). In relation with the first, it has been reported (see i.e [18]), that the critical field of some high $T_{c}$ superconductors diverges with decreasing $T$, which suggests the appearance of re-entering superconductivity in extremely strong magnetic fields. The vector component of this superconductor, according to our previous results, behave as ferromagnetic. Thus, for the 
superconductive phase in extremely strong magnetic fields we may expect the appearance of ferromagneticsuperconductive properties in those cases in which there is a significant amount of parallel spin paired fermions.

\section{Magnetization in the early universe}

We will consider the spontaneous magnetization of vector particles in connection with the condensation of $W^{ \pm}$ bosons, a phenomenon which was suggested by Linde [19] to occur in superdense matter, and by one of the present authors and Kalashnikov [21], to occur near the electroweak phase transition. The condensation of $W$ 's must be understood as some chemical equilibrium process, since the $W^{\prime}$ 's interacting with the quark background would decay in $n, p$ or $\pi^{ \pm}, \pi^{0}$ pairs.

If we consider at first the large density case, by taking $N_{W} \simeq N_{v c}$, where $N_{v c}=M_{W}^{3} c^{3} / 6 \pi^{2} \hbar^{3} \simeq$ $10^{45} \mathrm{~cm}^{-3}$, the magnetization would be of order $B \simeq$ $10^{19}$ Gauss. One may think as the mechanism of arising these huge fields as follows: the microscopic fields inside hadrons are of order $10^{15}$ Gauss. Such fields lead the $W$-condensate to magnetize, creating a field $10^{4}$ times larger. Then the conditions for spontaneous magnetization in domains arise. Obviously, $\mathcal{M}$ becomes very large (of order $B_{c}$ ) for fields near the critical value $B_{c} \simeq M_{W}^{2} c^{4} / e \hbar c \simeq 10^{24}$ gauss, since for $B \geq B_{c}$ the system becomes unstable leading to imaginary energy eigenvalues. [20]. Near the symmetry restoration temperature as $M=M(T)<M(0)$ the critical field occurs for smaller values of $B$.

The case of temperatures near the symmetry restoration lead to very interesting results. As shown in [21],[1], condensation of transverse vector $W$ bosons occurs for arbitrary small densities, and taking into account that the strong local magnetic fields would magnetize the condensate, we may think that (27) becomes singular, (for $M_{v}(T)=\sqrt{M_{W}^{2}(T)-e B \hbar / c^{3}} \rightarrow 0$, leading to infinite large magnetization of the medium, due to the vanishing of the (transverse, since the longitudinal modes acquire a Debye mass $\sim g T$ ) vector boson mass. One may think that this would lead to instabilities also, but we must remember that the zero mass of the $W_{s}$ for $T>T_{c}$ is only an approximate result first because actually the mass is expected to be of order $g^{2} T[22]$, second, because the self-magnetization $B=\mathcal{M}(B)$ that would arise, leads to a mechanism preventing the divergence to occur. In any case, the existence of a condensate in presence of strong hadron (or quark) fields may lead to the arising of a ferromagnetic behavior leading to extremely large fields, of order $B \leq B_{c}$, at temperatures $T \sim T_{c}$. The existence of such large fields of order $B_{c}$, as fluctuating fields on a scale $M_{W}^{-1}$, was first suggested by Vachaspati [23]. The same order has been estimated in [24] starting from the idea of some sort of equipartition of magnetic and radiation energies that may occur in a magnetically turbulent environment in the bubble formation arising from an electroweak first order phase transition.

\section{Conclusions}

We conclude that Bose-Einstein condensation of charged particles in a strong magnetic field is possible and leads to several new and interesting phenomena, as the occurrence of phase transition in presence of an external magnetic field, without a critical temperature. For low field intensity we have usual condensation, and for very strong fields, condensation is manifest again. The condensate in the strong magnetic field suggests the existence of superconductivity in extremely strong magnetic fields and the existence of a ferromagneticsuperconductive phase. This has interest in condensed matter physics. In astrophysics and cosmology we have also interesting consequences. It gives support to the conjectured existence of superfluid and superconductive phases in neutron stars [25]. It suggests also that at the electroweak phase transition, extremely strong magnetic fields may arise as a consequence of condensation and self-magnetization effects of the medium.

The authors would like to thank J. G. Hirsch for very important and detailed discussions and suggestions. They thank also R. Baquero and G. Gonzalez for fruitful comments. One of the authors (H.P.R.) thanks H. Rubinstein and G. Andrei Mezincescu for important discussions and M. Chaichian for hospitality in the High Energy Division of the Department of Physics, and acknowledges the financial support of the Academy of Finland under the Project No. 163394.

\section{References}

[1] M. Chaichian, R. Gonzalez Felipe, H. Perez Rojas, Phys. Lett. B 342, 245 (1995).

[2] M. R. Schafroth, Phys. Rev. 100, 463 (1955).

[3] R. M. May, J. Math. Phys. 6, 1462 (1965).

[4] J. Daicic, N. E. Frankel, and V. Kowalenko, Phys. Rev. Lett. 71, 1779 (1993).

[5] J. Daicic, N. E. Frankel, R. M. Gailis and V. Kowalenko, Phys. Rep.237, 63 (1994).

[6] D. J.Toms, Phys. Rev. Lett. 69, 1152 (1992); Phys. Rev. D 47, 2483 (1993); Phys. Lett. B 343, 259 (1995).

[7] P. Elmfors, P. Liljenberg, David Persson, Bo-Sture Skagerstam, Phys. Lett. B 348, 462 (1995).

[8] K. Kirsten and D. J. Toms, Phys. Rev. A 544188 (1996).

[9] K. Kirsten and D. J. Toms, Phys. Lett. B 368, 119 (1996).

[10] R. K. Pathria, Statistical Mechanics, Pergamon Press, Oxford, (1978). 
[11] Yu. Kagan, N.V. Prokof'ev and D.V. Svistunov, condmat/9908378

[12] L. D. Landau and E. M. Lifshitz, Statistical Physics, 3rd. Ed., Part 1, Pergamon Press (1986), New York.

[13] H. Perez Rojas, Phys. Lett. B 379, 148 (1996).

[14] H. Perez Rojas, Phys. Lett. A 234, 13 (1997).

[15] E.S. Fradkin, Proc. of the P.N. Lebedev Physical Inst. No. 29, Cons. Bureau (1967).

[16] H. Perez Rojas, Acta Phys. Pol. B 17, 861 (1986).

[17] G. A. Smolenski and V. A. Isupov, Sov. Journal of Techn. Phys.24, 1375 (1954); R. L. Moreira and R. P. S. M. Lobo, Jour. Phys. Soc. Japan 61, 1992 (1992).

[18] M. S. Osovski, R.J. Soulen Jr., S.A. Wolf, J.M. Broto, H. Rakoto, J.C. Oussel, G. Coffe, S. Askenazi, P. Pari, I. Bozovic, J.N. Eckstein and G. F. Virshup, Phys. Rev. Lett. 71, 2315 (1993); Yoichi Ando, G.S. Boebinger and
A. Passner, Phys. Rev. Lett 75, 4662 (1995); M. Rasolt and Z. Tesanovic, Rev. Mod. Phys. 64, 709 (1992).

[19] A. D. Linde, Phys. Lett. B 86 , 39 (1979).

[20] N.K. Nielsen and P. Olesen, Nucl. Phys. B 144, 376 (1978); J. Ambjorn, N.K. Nielsen and P. Olesen, Nucl. Phys. B 310, 625 (1988).

[21] O. K. Kalashnikov, H. Perez Rojas, Kratkie Soob. po Fizike, Lebedev Inst. Reports, Allerton Press 2, 2 (1986); H. Perez Rojas, O.K. Kalashnikov, Nucl. Phys. B 293, 241 (1987).

[22] A. D. Linde, Rep. Prog. Phys. 42, 389 (1979).

[23] T. Vachaspati, Phys. Lett. B 265, 258 (1991).

[24] G. Baym, G. Bodecker and D. Mc Lerran, Phys. Rev. D 53, 662 (1996).

[25] V. Thorsson, M. Prakash and J.M. Latimer, Nucl. Phys. A 572, 693 (1994). 